\title{
Automatic Engine Locking System Through Alcohol Detection
}

\author{
Dr. Pavan Shukla ${ }^{1}$, Utkarsh Srivastava $^{2}$, Sridhar Singh ${ }^{3}$, Rishabh Tripathi ${ }^{4}$, Rakesh Raushan Sharma ${ }^{5}$ \\ ${ }^{1}$ Co-Ordinator Dept. of E.C.E, RKGIT Ghaziabad \\ 2,3,4,5 Student , RKGIT Ghaziabad
}

\begin{abstract}
This project presents the design and implementation of an Alcohol Detection with Engine Locking for cars using the Ultrasonic Sensor and Arduino UNO as the MCU (Master Control Unit). The system will continuously monitor level of alcohol concentration in alcohol detection sensor and thus turn off the engine of vehicle if the alcohol concentration is above threshold level. The model will also send the message of whereabouts of the vehicle through SIM900A. The project provides an efficient solution to control accidents due to drunk driving.
\end{abstract}

Key Words: Arduino UNO, MQ-3 Sensor, Ultrasonic sensor, Buzzer, LED, SIM900A, DC Motor.

\section{INTRODUCTION}

The current scenario shows that the most of the road accidents are occurring due to drunk-driving. The drivers who drink alcohol are not in an stable condition and so, rash driving occurs on highway which can be risky to the lives of the people on road, the driver inclusive. The enormity of the dangerous driving transcends boundary. The laws in India are currently prohibiting drivers to drink and drive so that the fine can stop them to drink and drive. Whatsoever, effective observation of inebriated drivers could be a challenge to the policemen and road safety officers, the rationale for this stems from the natural inability of citizenry to be present additionally as state among identical house and time. This restricted ability of enforcement agents undermines each manual effort geared toward edge drink-driving. There is therefore the need for an alcohol detection system that can function without the restriction of space and time.

The Indian Ministry of Statistics reported thousands of road accidents in 2016. Though the report declared speed violation is the foremost reason for these accidents, it will safely be inferred that almost all of the cases are because of driver's unstable condition caused by drivers becoming drunk before they drive. The investigation done by the Planet Health Organization in 2008 shows that concerning 50\%-60\% of traffic accidents square measure associated with drink-driving. Moreover, WHO information on road traffic deaths disclosed 1.25 million traffic deaths were recorded globally in 2013 with the low- and middle-income countries having higher fatality rates per a $100 \mathrm{~K}$ population $(24.1 \%$ and $18.4 \%$ respectively), information collected showed that several of economic vehicles drivers in Bharat admitted to drinking alcohol throughout operating days. This shows that almost all drivers, particularly business and serious duty trucks drivers interact in drinkdriving, which may result in accident. Bharat sets a legal limit of $30 \mathrm{mg} / 100 \mathrm{~mL}$ blood alcohol concentration (BAC), any level higher than that's same to be ineligible. The BAC depicts the amount of alcohol in an exceedingly sure volume of blood. It's measured as either grams of alcohol per metric capacity unit of blood or milliliters of blood, $(\mathrm{mg} / \mathrm{ml}$, utilized in a lot of of Europe). For BAC level from 0.4 to 0.6 , drivers feel dazed/confused or otherwise disoriented, and it's typically not safe for a driver to drive a vehicle beneath such condition. Also, BAC level for 0.7 to 0.8 makes a driver's mental, physical and sensory functions to be severely impaired. At this stage, a driver is inactive and incapable of driving. BAC level of 0.2 to 0.3 continues to be not safe however the motive force still. So, there is need of such system which can reduce the number of road accidents caused due to drunk driving

\section{LITERATURE SURVEY}

A. The writer has put forward a technique which utilizes GPS and GSM to ascertain alcohol but this technique is very expensive,but the expenses can be cut off to a great extent. In this project a siren is being used which is highly economical, and can keep people in close proximity vigilant. [1]

B. Wearing smart helmet to prevent any mishap is suggested by writer which have certain deficiencies. Firstly restrictions on the use of helmets to only 2 wheelers. Secondly, microcontrollers are software based mega system in comparison to the economical siren that are open source hardware. [2]

C. Composite health monitoring and sensors based on infrared are utilized to ascertain alcohol as talked about by writer but the chance of false alarm can't be avoided in this system, because minute change in some situations can result in false alarm but in our project use of required technology makes it more authentic. [3]

D. To prevent the mishap of drunken driving writer have used PIC16F877A microcontroller which is an outdated system and expensive one also which restrains its use to only certain class of society whereas we are using Arduino and Uno microcontroller which is advanced as well as economical. [4]

E. Worrying about the drunken driving the writer suggests the system to overcome the issue but using mQ2 alcohol sensor has come flames .MQ2 alcohol sensor is not authentic and raises the chance of false alarm while we have used MQ3 which is highly authentic. [5] 
F. To cope with helmet negligence and alcohol detection simultaneous the writer proposed a system which is very complicated and use of P89V57RD2 microcontroller makes it highly expensive also this system can only be equipped with 2 wheelers whereas ,Aurdino uno microcontroller is economical as well as can be equipped with any class of vehicle making it more authentic and successful. [6]

\section{METHODOLOGY}

The Alcohol Detection with Engine Locking system helps to reduce accidents which are occurring due to drunk driving. MQ-3 sensor detects the presence of alcohol in the surroundings. The sensor provides output on the basis of the concentration of the alcohol, if the alcohol concentration is higher the conductivity of MQ-3 sensor increases which in turn gives the reading to ARDUINO.

If the reading is greater than the threshold level, ARDUINO will stop the DC motor. The red LED will also blink if the distance is less than the safe distance to give indication to other vehicles that the vehicle in front of them is unsafe. Now, with the help of SIM900A the message will be sent to the civil forces that the particular vehicle is unsafe and can be a threat to other people

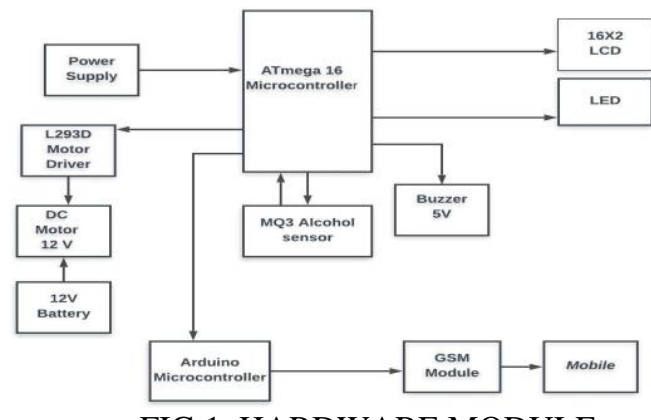

FIG 1. HARDWARE MODULE

\subsection{ARDUINO UNO}

R3Arduino Uno is micro-controller development board that works on Atmega-328P. The arduino results to numerous various functions like Microcontroller area unit, computer circuit unit, primarily little computers that will run tiny easy software package programs, the area unit are enough low steam-powered which can steam powered by various batteries for years however area unit ready to measure information much quicker than a person's brain can method/suppose.

The Board is an organization in the Italy which structures and sell-circuit sheets which make micro-controller simple to utilize, they coined those circuit sheets arduino and there will be a variety kinds of arduino, those can be utilized through variety of ways and has diverse usefulness of unique applications of instance we had straightforward arduinos sheets which seems like arduino-uno which can be conservative/very useful for each task.

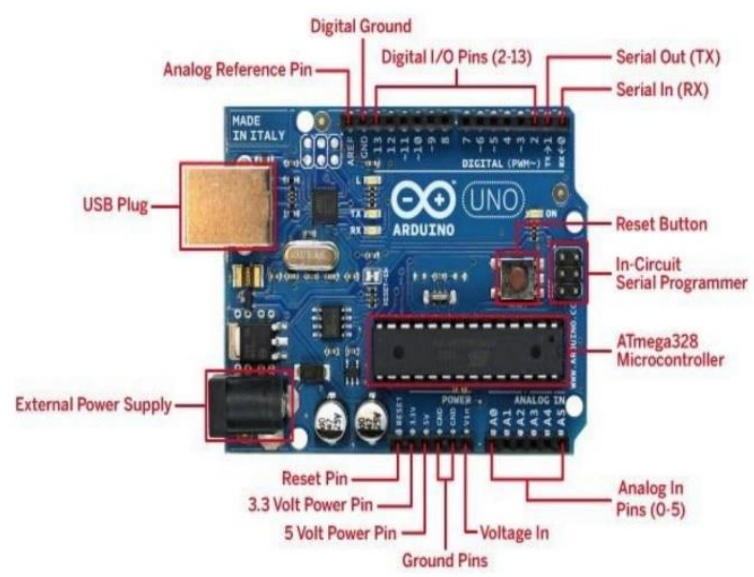

FIG 2. AURDUINO UNO

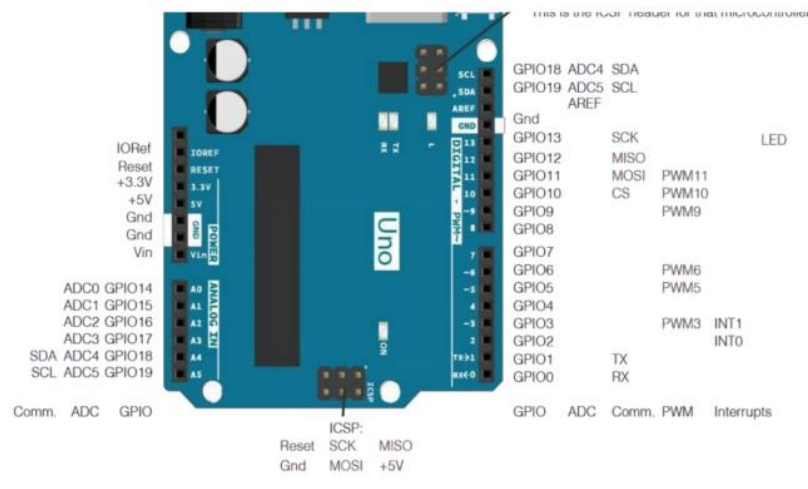

FIG 3. PIN DIAGRAM

\subsection{MQ-3 SENSOR}

The MQ-3 sensor is made of Tin Dioxide ( $\mathrm{SnO} 2)$ delicate layer. It is sorted out in such a structure to give high affectability to liquor and low affectability to Benzene. It has an immediate drive circuit to give lively reaction, quality, and longer lifetime. It is having a clear interface type. On the sensor, port pins 1, 2 and 3 tends to the yield, GND and VCC independently. The particular of the sensor is depicted in table underneath.

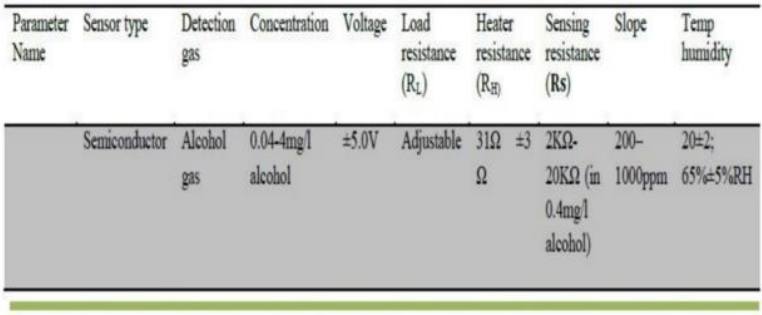

FIG 4. MQ3 SENSOR TABLE

The MQ-3 sensor is used to detect the presence of alcohol level in the surrounding and give reading to Arduino which determines whether the consumption level is in limit or not. 


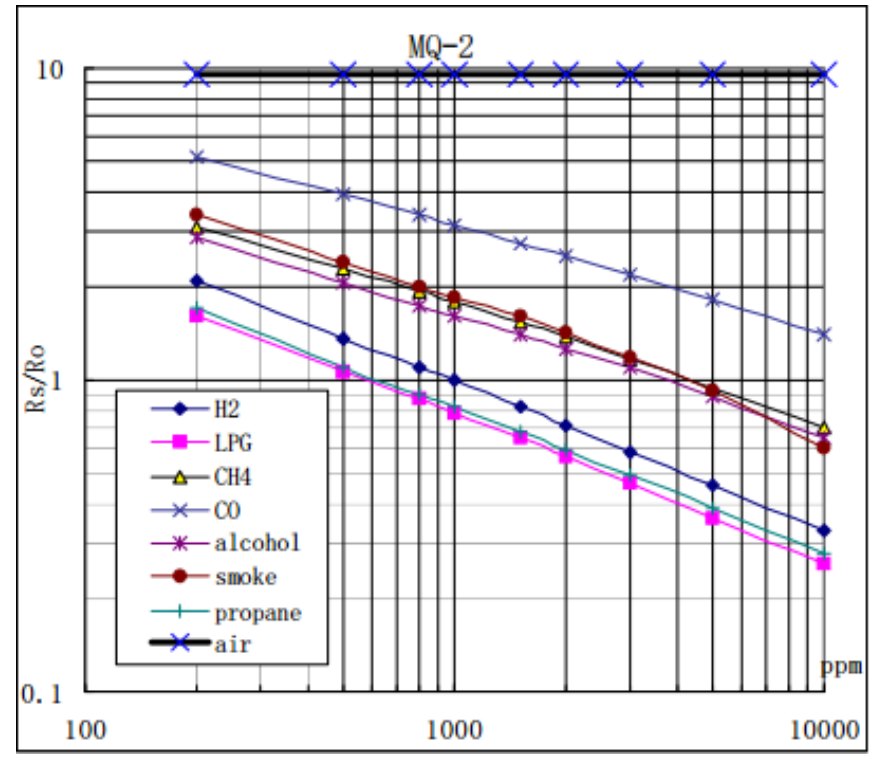

FIG 5. GAS SENSOR MODULE

\subsection{Buzzer}

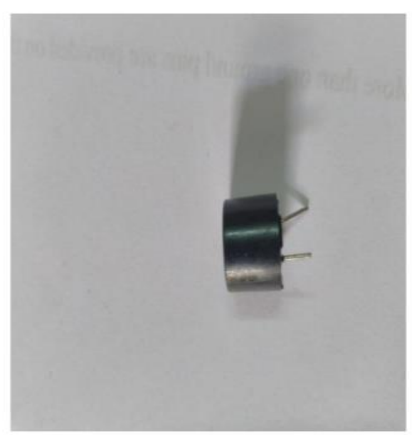

FIG 6. BUZZER

The alarm unit used is a buzzer which indicates when alcohol is detected. The buzzer is activated when an oscillating signal is passed through the coil of the buzzer and it fluctuates the disk present in the buzzer at a particular frequency which is equal to the driving signal. The buzzer indicates that vehicle in front of us is unsafe

\subsection{Motor}

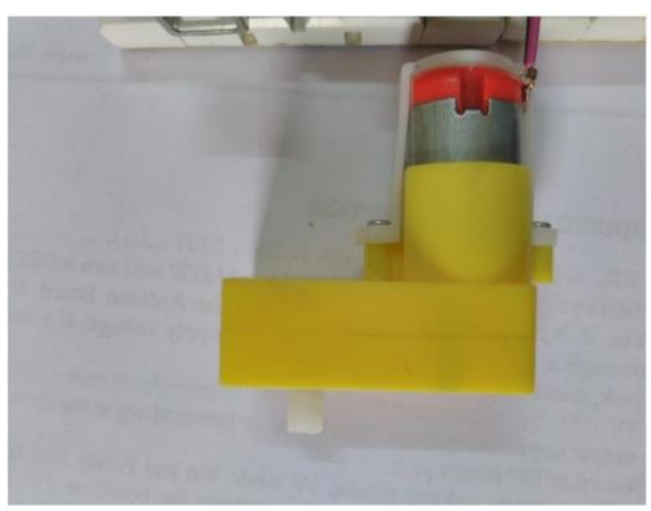

FIG 7 DC MOTOR

The DC motor is connected to the L293D which in turn is connected to Arduino and is given 5V supply. DC motor works on the principle of Lorentz Law. When an electric current is passed through the motor, the coil carrying the current produces magnetic field which in turn rotates the coil with the force experienced.

\subsection{SIM900A}

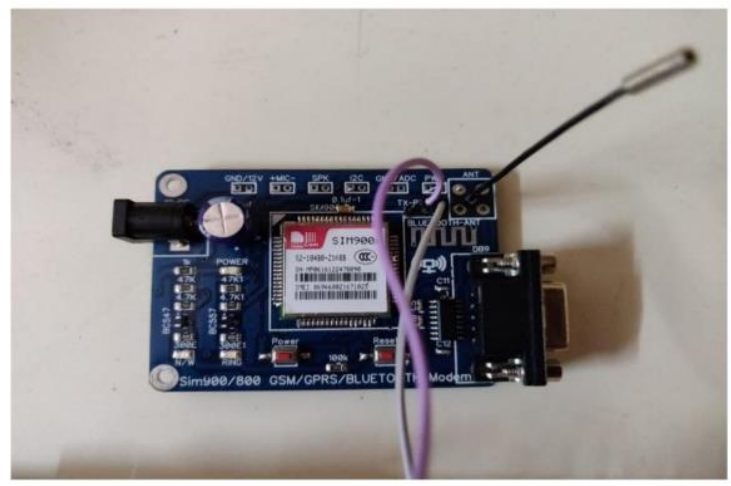

FIG 8. SIM900A

This is a smaller and solid remote module structure. The SIM900A is an entire group Dual-band GSM/GPRS strategy in a SMT module which can be installed in the client applications. The SIM is embedded in the SIM900A module and the message will individual that the vehicle is not safe.be sent to the cops or any specific

3.7 LED

An LED is a semiconductor light source which discharges centrality and oozes light the degree that photons when electrons join with electron openings. The shade of the light (standing out from the centrality of the photons) is compelled by the vitality required for electrons to cross the vitality band hole of the semiconductor and reach to conduction bands from the valence bands thus penetrating through energy gap.

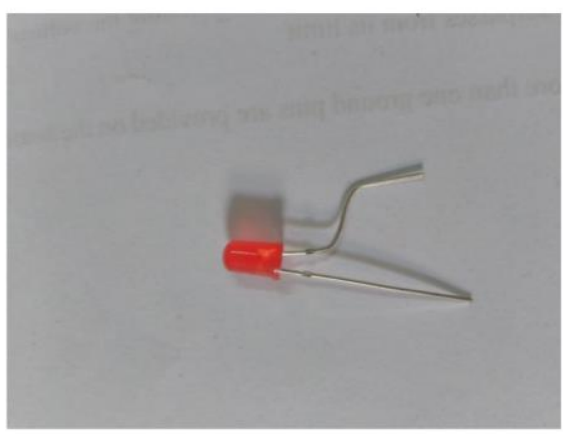

FIG 9.LED

\section{RESULT}

If alcoholic person tries command on vehicle the alcoholic sensor determines the existing of alcohol and shut down the vehicle engine and sound alarm by which the nearby people will exchange the seat. Peoples are aware of situation by the help of "LCD screen" present in the vehicles and hence take required action.We can avoided any kind of loss of life by using this system. All equipments are totally tested and connected as required thereby giving us the much needed result as shown in the image below 


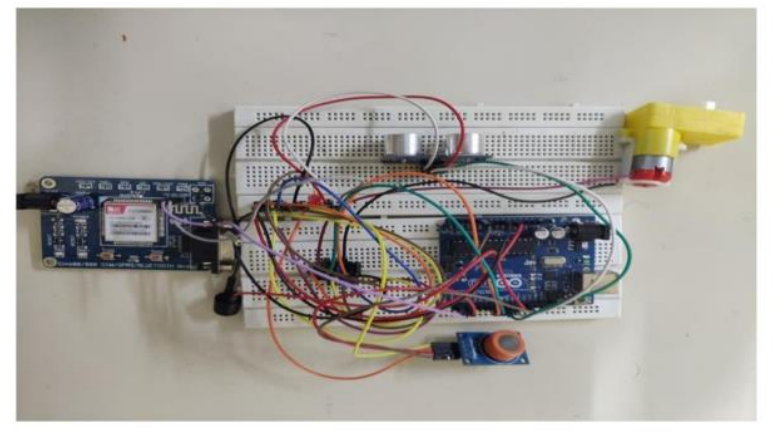

FIG 10. MODEL

\section{APPLICATIONS AND ADVANTAGES}

The applications of this project are easily visible.

- The Alcohol detection with engine locking system can be implemented in any $4-$ wheelers.

- The Alcohol detection with engine locking system can help prevent accidents due to drunk driving.

- The Alcohol detection with engine locking system can be very helpful for police.

- The Alcohol detection with engine locking system prove automatic safety system for cars and other vehicles.

\section{CONCLUSIONS}

We have given an incredibly capable way to deal and to develop a smart system for vehicles to diminish number of disasters caused in light of alcoholic driving. As the creating insight among people is that vehicle security is dynamically critical. Future degree of this structure is to control the setbacks caused due to alcohol use. This system improves the security of individual and in this manner giving the convincing progression in the vehicle business regarding decrease setbacks caused in light of driving.

\section{REFERENCES}

[1] L. A. Navarro, M. A. Diño, E. Joson, R. Anacan and R. D. Cruz, "Design of Alcohol Detection System for Car Users thru Iris Recognition Pattern Using Wavelet Transform," 2016 7th International Conference on Intelligent Systems, Modelling and Simulation (ISMS), Bangkok, 2016, pp. 15-19.

[2] Cahalan,D., I. Cisin, and Crossley, American Drinking Practices: A National Study of Driving Behaviour and Attitudes. 1969, Rutgers University Press: New Brunswick, NJ.

[3] MUGILA.G, MUTHULAKSHMI.M, SANTHIYA.K Prof.DHIVYA.P- SMART HELMET SYSTEM USING ALCOHOL DETECTION FOR VEHICLE PROTECTION[International Journal of Innovative Research in Science Engineering and Technology (IJIRTSE) ISSN: 2395-5619, Volume - 2, Issue - 7. July 2016].

[4] Dhivya M and Kathiravan S, Dept. of ECE, Kalaignar Karunanidhi Institute of Technology- Driver Authentication and Accident Avoidance System for Vehicles [Smart Computing Review, vol. 5, no. 1, February 2015].

[5] Babor, AUDIT: The alcohol use disorders identification Test: Guidelines for use in primary health care. 1992, Geneva, Switzerland: World Health Organization.

[6] Lee, Assessing the Feasibility of Vehicle-Based Sensors To Detect Alcohol Impairment. 2010, National Highway Traffic Safety Administration: Washington, DC.

[7] http://www.arduino.cc/

[8] A. ISuge, H.Takigawa, H.Osuga, H.Soma, K.Morisaki, Accident Vehicle Automatic Detection System By Image Processing Technology, OIEEE 1994 Vehiclee Navigation \& ISC.

[9] Paul Baskett, Yi Shang, Michael V. Patterson, Timothy Trull, Towards A System for Body-Area Sensing and Detection of Alcohol Craving and Mood Dvsregulation, (C) 2013 IEEE. 